\title{
Efficacy and safety of lenalidomide for refractory cutaneous lupus erythematosus
}

\author{
Josefina Cortés-Hernández, Gabriela Ávila, Miquel Vilardell-Tarrés and Josep Ordi-Ros
}

\begin{abstract}
Introduction: Cutaneous lupus erythematosus (CLE) is a chronic disease characterized by disfigurement and a relapsing course. Thalidomide has proven its efficacy in refractory cutaneous lupus disease, although it is not exempt from significant side effects and frequent relapses after withdrawal. New thalidomide analogues have been developed but lack clinical experience. The aim of this preliminary phase II study was to evaluate the efficacy and safety of lenalidomide in patients with refractory CLE.

Methods: Fifteen patients with refractory cutaneous lupus disease were enrolled in this single-center, open-label, non-comparative pilot trial between January 2009 and December 2010. Oral lenalidomide (5 to $10 \mathrm{mg} /$ day) was administered and tapered according to clinical response. Patients were followed up for a mean of 15 months (range: 7 to 30). Primary efficacy endpoint was the proportion of patients achieving complete response, defined by a Cutaneous Lupus Erythematosus Disease Area and Severity index (CLASI) activity score of 0. Other secondary endpoints included development of side effects, evaluation of cutaneous and systemic flares, and impact on the immunological parameters.

Results: One patient discontinued treatment due to side effects. All remaining patients saw clinical improvement and this was already noticeable after 2 weeks of treatment. Twelve of those patients (86\%) achieved complete response but clinical relapse was frequent (75\%), usually occurring 2 to 8 weeks after lenalidomide's withdrawal. No influence on systemic disease, immunological parameters or CLASI damage score was observed. Side effects including insomnia, grade 2 neutropenia and gastrointestinal symptoms, were minor (13\%). These resolved after withdrawing medication. Neither polyneuropathy nor thrombosis was observed.
\end{abstract}

Conclusion: Lenalidomide appears to be efficacious and safe in patients with refractory CLE, but clinical relapse is frequent after its withdrawal.

Trial registration: ClinicalTrials.gov: NCT01408199.

\section{Introduction}

Cutaneous involvement in lupus erythematosus disease is common, largely heterogeneous and characterized by a chronic relapsing course [1-3]. As many as 70 to $80 \%$ of patients will develop skin lesions at some point during the course of their disease, with a significant proportion being disfiguring and debilitating [1]. Although most patients respond to the standard first-line therapy of topical steroids and antimalarials, approximately 30 to $40 \%$ of cases will be refractory to these regimens. For this significant minority, there is no consensus algorithm and

\footnotetext{
* Correspondence: jordi@vhebron.net

Medicine Department, Systemic Autoimmune Diseases Unit, Hospital Universitari Vall d'Hebron, Institut de Recerca (VHIR), Universitat Autònoma de Barcelona, Passeig Vall d'Hebron 119-129, 08035 Barcelona, Spain
}

a trial and error approach using multiple systemic agents has shown a variable poor response [4-6].

Thalidomide, a glutamic acid derivative, is an agent with a mechanism of action that includes both tumoricidal and immunomodulatory effects. It was first synthesized in 1954 and widely used as a sedative agent, but had to be withdrawn from the market after teratogenic and neurologic effects were established [7]. In recent years, there has been a renewed interest in its use, and currently is also prescribed in various oncologic, dermatologic, and inflammatory conditions, including refractory cutaneous lupus erythematosus (CLE) [8]. In the latter, a significant rapid clinical response has been reported in 80 to $90 \%$ of the treated patients [8-17]. However, despite its effectiveness,

\section{() Biomed Central}


sustained long-term remission after its withdrawal is low, relapse occurs frequently and it is not exempt from severe side effects that are the main limitation on its continued use [17].

Given thalidomide's vast clinical potential to treat inflammatory and neoplastic conditions, efforts in the mid-1990s led to the development of a promising thalidomide analogue, lenalidomide (CC-5013 or Revlimid ${ }^{\mathrm{TM}}$ ), to improve tolerability and efficacy over the parent drug. In recent years, lenalidomide ( 5 to $50 \mathrm{mg} /$ day) has been most extensively studied and approved for refractory/ relapsing multiple myeloma and myelodysplastic syndromes [18,19].

Thalidomide and its immunomodulatory analogues (IMiDs), first established as agents with antiangiogenic properties, inhibit the production of cytokines (TNF- $\alpha$ ), interleukins (IL) $1 \beta, 6,12$ and TNF- $\alpha$-induced cell surface adhesion molecules (ICAM-1, VCAM-1, E-selectin). In addition, they promote apoptosis, and increase the NK-dependent cytotoxicity by altering natural killer (NK) cell numbers and function [20]. Although both IMiDs have demonstrated similar biological activities, lenalidomide is more potent than thalidomide and therefore achieves responses at lower doses [20-22]. Lenalidomide has also been shown to have a different toxicity profile. Whereas somnolence, constipation and peripheral neuropathy have been less reported [21], myelosuppression occurs more frequently in a dose-independent manner [18,20-22].

Thus, based on its immunomodulatory properties, the favorable toxicity profile and its potential superior efficacy compared to the parent drug, lenalidomide may be an alternative in refractory inflammatory dermatosis such as cutaneous lupus. So far, there is little familiarity with its use in this field and only a small number of patients have been treated with lenalidomide ( 5 to $10 \mathrm{mg} /$ day) albeit with encouraging results $[23,24]$. Given the limited clinical experience, its potential beneficial effects and the need to better understand its effect on the systemic facet of the lupus disease, a larger open phase II trial was initiated.

\section{Materials and methods Study design}

The purpose of this open-labelled, single centre, phase II pilot study was to establish the efficacy and safety of lenalidomide in refractory CLE. The study protocol was approved by the local research ethics committee of Vall d'Hebron Hospital and was conducted according to $\mathrm{ICH}$ good clinical practice and in accordance with the Declaration of Helsinki. All patients gave their written informed consent. The study was registered at the ClinicalTrials.gov (Identifier: NCT01408199).

\section{Endpoints}

The efficacy primary endpoint was the proportion of patients achieving complete response. Clinical response was evaluated by the validated Cutaneous Lupus Erythematosus Disease Area and Severity Index (CLASI) [25]. Response was defined as follows: complete response (CR) as a complete resolution of the inflammatory rash (CLASI activity score $=0)$; partial response $(\mathrm{PR})$ by at least a $50 \%$ improvement in the CLASI score by week 12 when compared to baseline, and no response when no improvement or worsening in the CLASI score was observed at the same time period.

Secondary endpoints included the proportion of patients: developing any adverse event (AE); having a cutaneous relapse, defined as a new CLASI $\geq 4$ in those who previously achieved CR following treatment withdrawal; developing a systemic lupus flare, assessed by the Systemic Lupus Erythematosus Disease Activity Index 2000 (SLEDAI) [26] in which a mild flare was defined as a change in SLEDAI score greater than 3 and a severe flare by a change in SLEDAI greater than 12; having an increment in the CLASI damage score [25] compared to baseline or having an increment of anti-dsDNA antibody titres.

An $\mathrm{AE}$ was defined as any adverse deviation from the patient's baseline condition during the trial. The events were categorised as mild, moderate or severe. A serious AE (SAE) was defined as an event that was life-threatening, resulted in death, required or prolonged hospitalisation or resulted in persistent or significant disability/incapacity.

Reasons for discontinuing treatment were: common toxicity criteria grade 3 myelosuppression, withdrawal of consent by the patient, pregnancy, life-threatening complications, severe systemic lupus flare requiring alternative treatment or decision by the physician that discontinuation was in the patient's best interest.

\section{Inclusion criteria}

Patients were recruited from a single centre between Jan 2009 and 2010 according to the following eligibility criteria: aged over 18 years; histological proven CLE with or without associated systemic lupus erythematosus (SLE) disease diagnosed according to the American College of Rheumatology (ACR) SLE classification criteria [27]; presence of at least a grade II erythema as assessed by the CLASI activity score [25]; stable prednisone (<10 mg/day), antimalarial, or immunosuppressive regimens for at least 30 days before the inclusion; refractoriness to at least 3 months of conventional treatment with antimalarials and topical steroids; and finally, at least one of, an involvement of more than $18 \%$ of the body surface area calculated according to the 'rule of the nines', a history of severe thalidomide side effects or lack of efficacy following thalidomide therapy. 
For all patients, there was no known hypersensitivity to thalidomide. Women were excluded from the study if pregnant, lactating or not using adequate contraception. Other key exclusion criteria included active SLE requiring systemic immunosuppressive agents, presence of severe thrombocytopenia $\left(<30,000 / \mathrm{mm}^{3}\right)$, leukopenia $(<2,000 /$ $\mathrm{mm}^{3}$ ) or neutrophil counts below $1,000 / \mu \mathrm{l}$, known at least 30 days prior to inclusion; previous history of arterial/ venous thrombosis; presence of antiphospholipid antibodies; moderate to severe renal impairment (FG $<30 \mathrm{ml} /$ $\min / 1.73 \mathrm{~m}^{2}$ ) and/or progressive renal disease. Psychiatric or social disorder that might interfere with the follow-up or treatment compliance, HIV or viral hepatitis infections were also exclusion criteria. All patients gave written informed consent prior to participation in the study.

\section{Treatment protocol and assessment}

Lenalidomide was started at $5 \mathrm{mg} /$ day for 4 weeks. At that time, if no clinical improvement was observed, using the criteria specified before, dose was increased to $10 \mathrm{mg} /$ day. Otherwise, lenalidomide was sustained at $5 \mathrm{mg} /$ day in case of partial response or decreased progressively monthly until its withdrawal if complete response was achieved. No changes in concomitant baseline medication were allowed.

At entry, patients had a complete medical history review and physical examination, including a detailed neurological assessment. At baseline measurements of full blood counts and immunological parameters (antidsDNA, complement, antinuclear antibodies (ANA), anti-extractable nuclear antigen (ENA) and antiphospholipid antibodies) were performed. The evaluation of each patient's rash took into account the initial extension, activity and degree of scarring as assessed by the CLASI score [25]. This score consists of two separate scores: the activity score, which reflects erythema and scaling; and the damage score, which documents scarring and permanent dyspigmentation.

Patients were followed-up monthly for the first 6 months and every three months afterwards, unless clinically required. At each visit, clinical parameters including a detailed neurological examination, the CLASI score, response to therapy, and occurrence of side effects were evaluated. Nerve conduction studies were performed in those patients with previous thalidomide-induced neuropathy or when new neurological symptoms occurred. Laboratory tests (WBC) were performed weekly for the first 2 months and then monthly following drug safety recommendations. Exacerbations of systemic disease during follow-up were evaluated by the SLEDAI score [26].

\section{Statistical analysis}

Statistical analysis was performed using SPSS version 7.5 (SPSS, Inc., Chicago, IL, USA). Descriptive statistics are reported as frequency and percentage for categorical variables and as mean and standard deviation for continuous variables. Categorical and continuous data were compared by means of the $t$ test and Mann-Whitney test, as appropriate. The level of statistical significance was set at $P<0.05$.

\section{Results}

\section{Baseline patient characteristics}

Fifteen consecutive patients with refractory CLE were included. The major subtypes of the recruits were discoid lupus erythematosus (DLE) (60\%), lupus profundus (13\%) and subacute cutaneous lupus erythematosus (SCLE) (13\%). Table 1 shows baseline clinical and serological data. Fourteen of them had been previously treated with thalidomide and either did not respond $(n=4)$ and/or developed severe side effects $(n=11)$. Four patients also had a rash covering more than $18 \%$ of the body surface area. All patients were female and of Caucasian origin. Age varied between 28 and 53 with a median age of 40 years. Six patients $(40 \%)$ had an associated systemic disease, of whom five had positive anti-dsDNA antibodies. Photosensitivity was present in seven patients (47\%). The mean $( \pm$ SD) CLASI activity and damage scores at entry were $11 \pm 5.9$ and $2.33 \pm 2.96$, respectively. Initial SLEDAI score was $3.26 \pm 2.22$.

\section{Clinical efficacy}

Clinical response and lenalidomide dosage are shown in Table 2 and Figure 1. One patient discontinued the study after 1 week due to disabling gastrointestinal side effects (patient number 3). Clinical response (combined complete and partial response) was observed in all of the remaining patients (100\%) (Figure 1), of whom 12 (86\%) achieved a CR. Four of the responders had previously failed thalidomide therapy. A significant clinical improvement was already noticeable at 2 weeks (CLASI activity decreased from $11 \pm 5.9$ to $4.13 \pm 3.66, P=0.0009$ ). Time to CR in those patients with DLE and SCLE was shorter and ranged from 2 to 12 weeks with a median time of 6 weeks. However, in those with lupus profundus, time to CR varied from 4 to 32 weeks with a median time of 13 weeks. At the end of follow-up, two patients (14\%) remained in partial response, despite increasing the dose in one case (10 mg/day). Overall, treatment duration ranged from 3 to 30 months with a median of 11 months.

Patients were followed up for a median of 15 months (range: 7 to 30). Of the twelve patients who achieved complete response, nine (75\%) relapsed within 2 to 8 weeks (median 4.4), while reducing medication or after its withdrawal. The lowest dose of lenalidomide at which relapse occurred was $5 \mathrm{mg}$ on alternate days or three times weekly. Five patients had at least two relapses when 
Table 1 Patient demographics and clinical characteristics.

\begin{tabular}{|c|c|c|c|c|c|c|c|c|}
\hline $\begin{array}{l}\text { Patient } \\
\text { number }\end{array}$ & Sex & Diagnosis & $\begin{array}{c}\text { Site } \\
\text { involvement }\end{array}$ & Reason for inclusión & $\begin{array}{c}\text { ANA/dsDNA }^{\alpha} \\
\text { antibodies }\end{array}$ & $\begin{array}{c}\text { Anti-Ro/SSA/La/SSB } \\
\text { antibodies }\end{array}$ & $\begin{array}{l}\text { CLASI } \\
\text { A/D }\end{array}$ & $\begin{array}{c}\text { Previous received } \\
\text { treatment }\end{array}$ \\
\hline 1 & $F$ & DLE (L) & V-neck, arm & T lack of efficacy, PN & $1 / 640(123)$ & $+/-$ & $6 / 2$ & TCS, OCS, HCQ, T \\
\hline 2 & $\mathrm{~F}$ & $\begin{array}{l}\text { Lupus } \\
\text { Profundus }\end{array}$ & Arm, back & T lack of efficacy & $1 / 160(-)$ & $-/$ & $7 / 1$ & TCS, OCS, HCQ, T \\
\hline 3 & $\mathrm{~F}$ & DLE (L) & Arm & T lack of efficacy & $1 / 640(-)$ & $-/$ & $3 / 1$ & TCS, OCS, HCQ, T \\
\hline 4 & $\mathrm{~F}$ & ACLE & Face, v-neck & $T$ side effects & $1 / 640(54)$ & $-/$ & $6 / 0$ & $\begin{array}{c}\text { TCS, OCS, HCQ, T, } \\
\text { AZA, }\end{array}$ \\
\hline 5 & $\mathrm{~F}$ & $\begin{array}{l}\text { Lupus } \\
\text { Profundus }\end{array}$ & Scalp, face, arms & $T$ side effects & $1 / 640(144)$ & $-/$ & $19 / 6$ & $\begin{array}{c}\text { TCS, OCS, HCQ, } \\
\text { MMF, T }\end{array}$ \\
\hline 6 & $\mathrm{~F}$ & SCLE & $\begin{array}{l}\text { Face, v-neck, } \\
\text { back }\end{array}$ & $>18 \%$ body surface & $1 / 640(-)$ & $+/-$ & $19 / 0$ & TCS, OCS, MMF \\
\hline 7 & F & SCLE & $\begin{array}{l}\text { Face, v-neck, } \\
\text { hands, arms }\end{array}$ & $\begin{array}{c}\text { T side effects, } \mathrm{PN},>18 \% \\
\text { body surface }\end{array}$ & $1 / 640(83)$ & $-/$ & $20 / 1$ & $\begin{array}{c}\text { TCS, OCS, } \mathrm{HCQ} \\
\mathrm{AZA}, \mathrm{T}\end{array}$ \\
\hline 8 & F & DLE (L) & $\begin{array}{l}\text { Face, scalp, ear } \\
\text { lobe }\end{array}$ & $T$ side effects & $1 / 40(-)$ & $-/$ & $9 / 0$ & TCS, OCS, HCQ, T \\
\hline 9 & $\mathrm{~F}$ & $\operatorname{DLE}(\mathrm{G})$ & $\begin{array}{l}\text { Face, ear lobe, } \\
\text { arms, neck }\end{array}$ & $\begin{array}{c}>18 \% \text { body surface, } T \\
\text { side effects }\end{array}$ & $1 / 320(37)$ & $-/-$ & $13 / 2$ & $\begin{array}{c}\text { TCS, OCS, HCQ, } \\
\text { MMF, T }\end{array}$ \\
\hline 10 & $\mathrm{~F}$ & LET & Face & T side effects, PN & $1 / 640(-)$ & $-/-$ & $6 / 0$ & $\mathrm{TCS}, \mathrm{HCQ}, \mathrm{T}$ \\
\hline 11 & $\mathrm{~F}$ & $\operatorname{DLE}(G)$ & Face, scalp, back & $\begin{array}{l}\text { T side effects, }>18 \% \\
\text { body surface }\end{array}$ & $1 / 40(-)$ & $+/-$ & $15 / 9$ & TCS, OCS, HCQ, T \\
\hline 12 & $\mathrm{~F}$ & $\operatorname{DLE}(\mathrm{G})$ & $\begin{array}{l}\text { Face, v-neck, } \\
\text { back, scalp }\end{array}$ & T side effects, PN & $1 / 40(-)$ & $+/-$ & $17 / 8$ & TCS, OCS, HCQ, T \\
\hline 13 & $\mathrm{~F}$ & DLE (L) & Face & T lack of efficacy & $1 / 160(-)$ & $-/$ & $3 / 0$ & TCS, HCQ, T \\
\hline 14 & $\mathrm{~F}$ & $\operatorname{DLE}(\mathrm{G})$ & Face, v-neck & $T$ side effects & $1 / 640(-)$ & $-/-$ & $11 / 2$ & $\begin{array}{c}\text { TCS, OCS, HCQ, } \\
\text { MMF, T }\end{array}$ \\
\hline 15 & $\mathrm{~F}$ & DLE (L) & Face, scalp & $T$ side effects & $1 / 40(-)$ & $-/-$ & $10 / 3$ & TCS, OCS, HCQ, T \\
\hline
\end{tabular}

${ }^{\alpha}$ Normal value of anti-dsDNA antibodies is < $15 \mathrm{lU} / \mathrm{ml}$. A, activity; ACLE, acute cutaneous lupus erythematosus; ANA, antinuclear antibodies; AZA, azathioprine; CLASI, Cutaneous Lupus Erythematosus Disease Area and Severity Index; D, damage; DLE, discoid lupus erythematosus; G, generalized; HCQ, hydroxychloroquine; L, localized; LET, lupus erythematosus tumidus; MMF, mycophenolate mofetil; OCS, oral corticosteroids; PN, polyneuropathy; SCLE, subacute cutaneous lupus erythematosus; T, thalidomide; TCS, topical steroids.

Table 2 Summary of clinical response.

\begin{tabular}{|c|c|c|c|c|c|c|}
\hline $\begin{array}{l}\text { Patient } \\
\text { number }\end{array}$ & $\begin{array}{l}\text { Clinical } \\
\text { outcome }\end{array}$ & $\begin{array}{l}\text { Time to complete response } \\
\text { (weeks) }\end{array}$ & $\begin{array}{l}\text { Follow-up period } \\
\text { (months) }\end{array}$ & $\begin{array}{l}\text { Maximun dose received } \\
(\mathrm{mg})\end{array}$ & Relapse & Dose at relapse \\
\hline 1 & $C R$ & 10 & 15 & 5 & Yes & $5 \mathrm{mg}$ twice weekly \\
\hline 2 & $C R$ & 4 & 12 & 5 & Yes & $\begin{array}{l}5 \mathrm{mg} \text { three times } \\
\text { weekly }\end{array}$ \\
\hline 3 & Withdrawn & - & - & 5 & - & - \\
\hline 4 & $C R$ & 3 & 12 & 5 & Yes & $5 \mathrm{mg}$ alternate days \\
\hline 5 & $C R$ & 36 & 19 & 5 & - & - \\
\hline 6 & $C R$ & 4 & 17 & 5 & No & - \\
\hline 7 & $C R$ & 11 & 18 & 5 & No & - \\
\hline 8 & $C R$ & 2 & 7 & 5 & Yes & $5 \mathrm{mg}$ alternate days \\
\hline 9 & $P R$ & - & 15 & 10 & - & - \\
\hline 10 & $C R$ & 7 & 10 & 5 & Yes & $5 \mathrm{mg}$ alternate days \\
\hline 11 & $C R$ & 4 & 30 & 5 & Yes & 5 mg twice weekly \\
\hline 12 & $C R$ & 4 & 25 & 5 & Yes & $5 \mathrm{mg}$ alternate days \\
\hline 13 & $P R$ & - & 8 & 5 & - & - \\
\hline 14 & $C R$ & 12 & 17 & 5 & Yes & $\begin{array}{l}5 \mathrm{mg} \text { three times } \\
\text { weekly }\end{array}$ \\
\hline 15 & $C R$ & 2 & 10 & 5 & Yes & $5 \mathrm{mg}$ alternate days \\
\hline
\end{tabular}

$\mathrm{CR}$, complete response; PR, partial response. 
A.

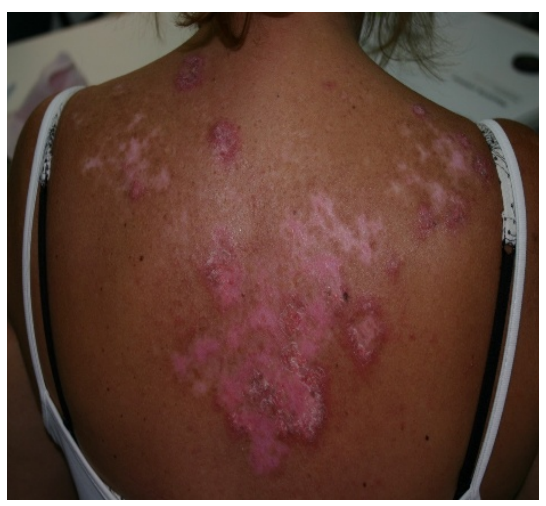

C.

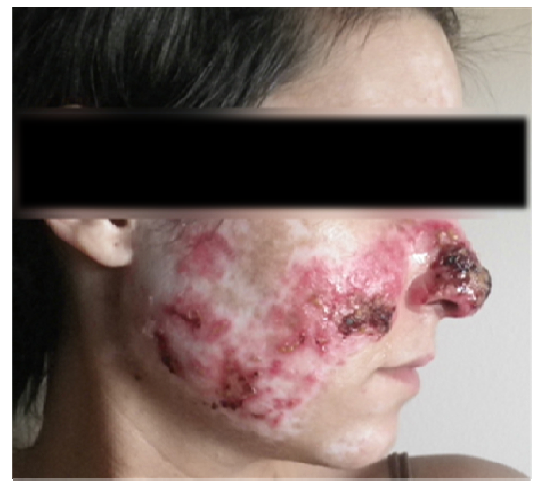

E.

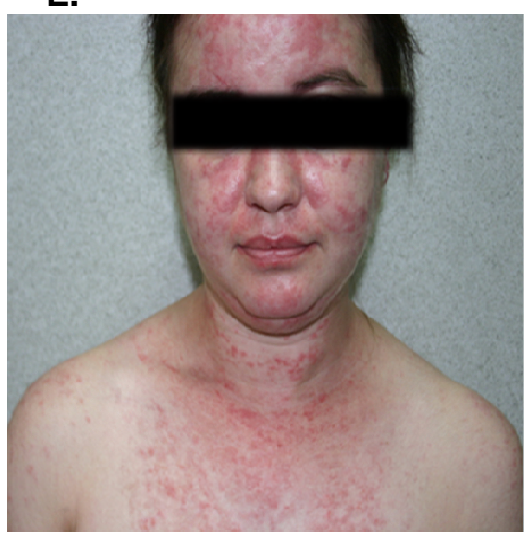

B.

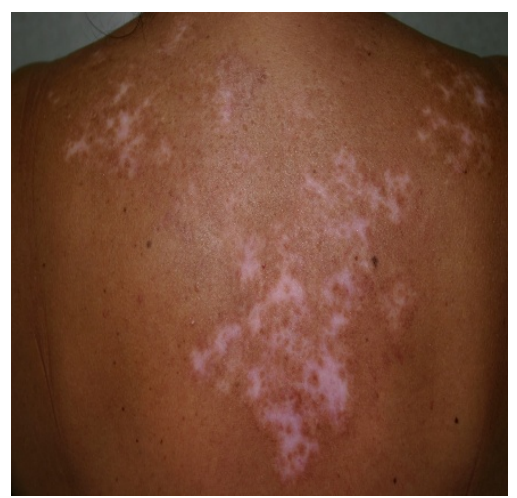

D.

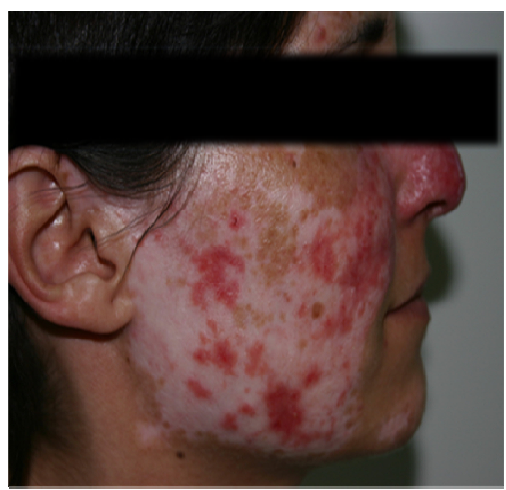

$\mathbf{F}$.

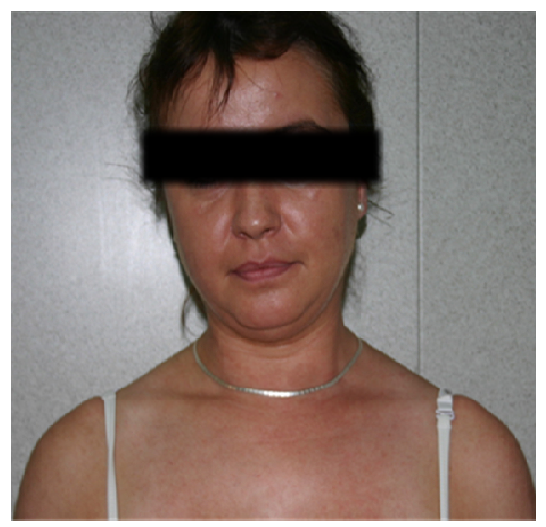

Figure 1 Clinical responses to lenalidomide therapy. Discoid lupus erythematosus (patient number 12) on upper back before (A) and after 3 months of lenalidomide therapy ( $5 \mathrm{mg} / \mathrm{d}$ ) (B). Resolution of the erythema with persistent residual hypopigmentation and some degree of scarring, already existing at baseline from previous cutaneous involvement. Widespread discoid lupus erythematosus (DLE) (patient number 9) involving nose and malar area before (C) and after three months of therapy (D) resulting in a partial improvement of the erythema and increased hypopigmentation. Widespread subacute cutaneous lupus erythematosus (SCLE) (patient number 6) involving face and V-neck area before $\mathbf{( E )}$, and complete resolution after 6 weeks of lenalidomide therapy $\mathbf{F}$ ). All patients concerned gave their written consent for the publication of the images. 
reducing dose and received two cycles of treatment. All patients responded to the introduction of lenalidomide. Three of the fourteen treated patients (21\%) required a long-term maintenance dose. Only two patients, both with SCLE, obtained a sustained remission after withdrawing medication.

After one year follow-up, although a slight increment in the CLASI damage score was observed compared with baseline ( $3.08 \pm 3.15$ vs. $2.39 \pm 2.96$, respectively, $P=$ 0.398 ), this was not statistically significant (Figure 2).

\section{Effect on systemic disease}

Clinically, only one of the seven patients (14\%) with associated SLE developed arthralgias and mild arthritis during follow-up (patient number 1). None of the patients with only cutaneous lupus developed systemic symptoms. No significant changes in the mean SLEDAI score were observed during follow-up. Two patients had previous history of lupus nephritis. No new onset or increase in proteinuria was observed. When the impact of lenalidomide was evaluated on the immunological parameters, anti-dsDNA titres did not experience significant changes in patients with or without previous anti-dsDNA antibodies. No effect was observed in complement levels (C3 and $\mathrm{C} 4$ ) either (Figure 3).

\section{Toxicity}

Generally, lenalidomide was well tolerated. Only two patients developed side effects (13\%) (Table 3). Patient number 3 , one week after medication was initiated,

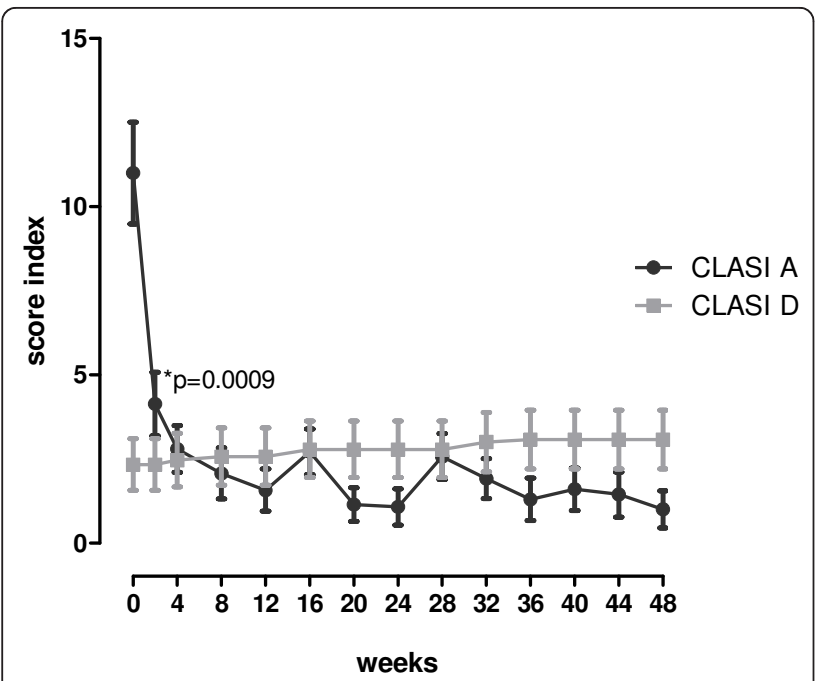

Figure 2 Cutaneous Lupus Erythematosus Disease Area and Severity Index (CLASI). CLASI shows a reduction of the CLASI activity (CLASI A $(\bullet)$ ) score with no significant changes in the CLASI damage (CLASI D (-)) score over 12 months of follow-up. A significant improvement of the inflammatory rash was already observed by week 2. Data is expressed as mean \pm SEM. ${ }^{*} P$ value refers to the comparison of CLASI scores between two weeks after treatment and baseline. developed severe vomiting, diarrhoea and weight loss that improved after medication was withdrawn and no other factors accounting for this episode were found. Patient number 15 developed insomnia and grade 2 neutropenia $\left(33 \%\right.$ neutrophils $\left.\left(1.190 / \mathrm{mm}^{3}\right)\right), 2$ months into the study while on lenalidomide $5 \mathrm{mg} /$ day. There was no evidence that somnolence was a problem for patients taking the study drug. None of the patients complained of paraesthesia. A repeated electrophysiological study in those patients with previously known thalidomideinduced neuropathy was performed. No worsening was observed. No other evidence of haematological or biochemical toxicities was observed. There were no thrombotic events or ovarian toxicity during the study.

\section{Discussion}

The purpose of this trial was to investigate the efficacy and safety of lenalidomide, a new thalidomide analogue, in the treatment of refractory CLE. Although in recent years there has been a better understanding of the pathophysiological mechanisms involved in the development of CLE, there has not been a great breakthrough in its treatment. So far, no medication has been approved specifically for this condition and few randomized, placebocontrolled trials, evaluating mainly topical agents in patients with DLE, have been performed [28-30]. Currently, the first-line therapy consists of antimalarials and topical steroids, but for refractory cases there is no consensus, and in most cases, off-label systemic agents licensed for SLE and other immunological diseases are administered.

Thalidomide is one of the few agents with proven efficacy for refractory CLE according to case reports and observational studies [9-17]. However, despite its effectiveness, its use has been limited due to its toxicity profile and the current restricted availability. This trial was encouraged by the partial beneficial effects initially published in two patients with recalcitrant DLE who had been treated with lenalidomide (5 to $10 \mathrm{mg} /$ day) [23] and by our experience using thalidomide in this condition $[13,17]$. After excluding the patient with disabling side effects, we included a total of 14 patients with refractory CLE. All patients experienced clinical improvement. Complete response was achieved by $86 \%$ of them within 2 to 12 weeks after starting lenalidomide. This rate of CR is different from the only report published to date using lenalidomide in CLE. Braunstein $e t$ al. [24] described a small cohort of five patients treated with the same regimen for 6 weeks. At the end of treatment, in that study, the majority of patients (four out of five) achieved a partial response defined by at least a 4point decrease in the CLASI score, although most of them experienced a decrease of at least 8 points [24]. Differences in treatment duration, clinical response 


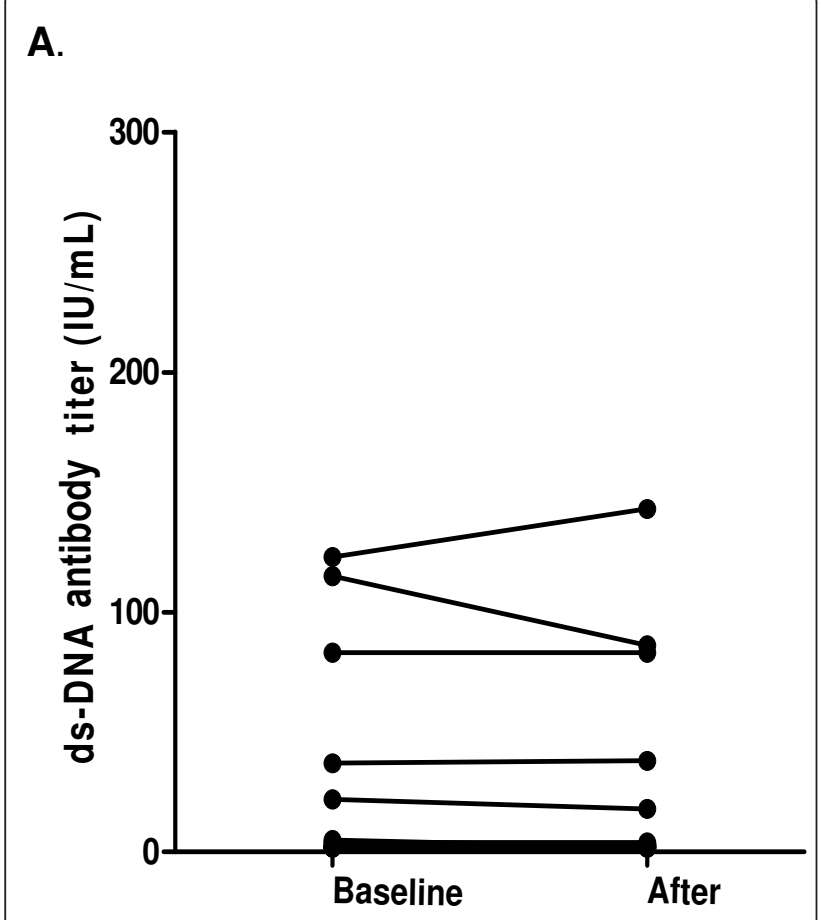

B.

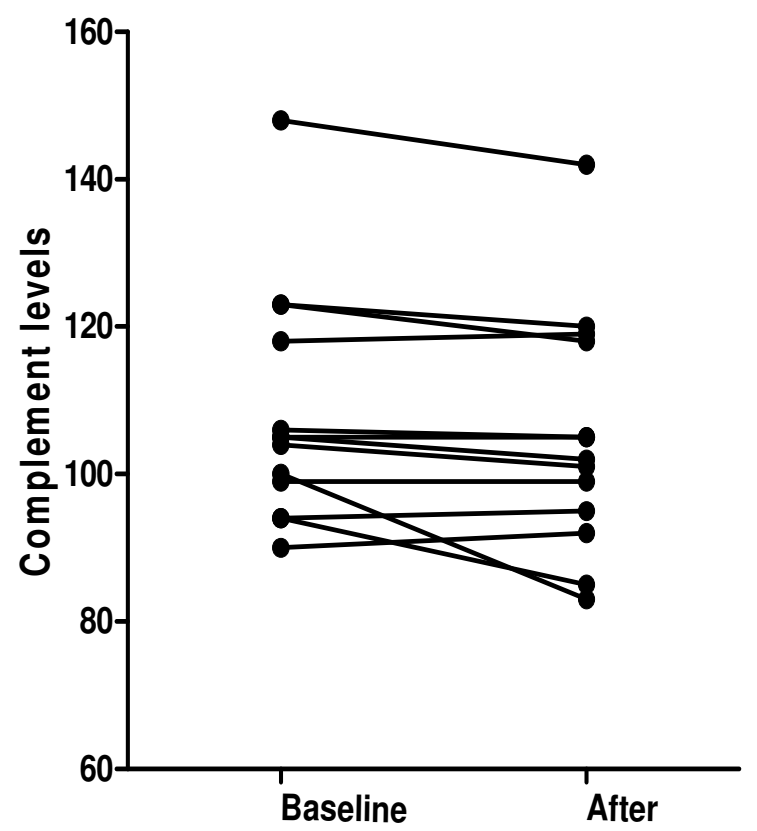

Figure 3 Effects of Lenalidomide on anti-dsDNA antibody and complement levels. Comparison of anti-dsDNA antibody titres (A) and complement levels (B) at baseline and after completing the course of treatment. definition and follow-up between the two studies account for the discrepancies observed. Interestingly, this study also established for the first time preliminary histological and biological factors associated with lenalidomide clinical response. Whereas nonresponders had high CXCL10 cutaneous expression and increased activated circulating $\mathrm{CD} 4+\mathrm{T}$ cells, responders had reduced glycosaminoglycan and pDCs content with a trend toward increased circulating $\mathrm{T}$ regulatory cells. No correlation with the IFN signature was established [24].

As with thalidomide [17], cutaneous relapse was frequent and occurred in $75 \%$ of patients within 2 to 8 weeks after tapering dosage or withdrawing medication. Although the study was not designed to establish variations in clinical response between the different histological subtypes of CLE and few patients were included, it was observed that whereas patients with refractory SCLE tended to remain in remission after withdrawing medication, those with DLE or lupus erythematosus tumidus tended to relapse more frequently. Some of the relapsing patients (21\%) even required a low long-term maintenance dose $(5 \mathrm{mg} /$ alternate days or three times a week) to control the disease.

In the literature to date, a variety of adverse effects have been reported, mainly in patients with haematological conditions and receiving higher doses of lenalidomide. The most commonly described include neutropenia, thrombocytopenia, paraesthesia, skin and gastrointestinal toxicity, and fatigue. Overall, myelosuppression, generally mild, has been the most common, and although is more frequently observed at high doses ( 25 to $50 \mathrm{mg} /$ day), it can occur in a dose-independent manner $[18,22,23,31,32]$. In our study, lenalidomide was generally well tolerated and at this dosage displayed a safe profile. The few observed side effects resolved after drug withdrawal. No neurological symptoms were reported and none of the patients with previous thalidomide-induced polyneuropathy experienced worsening in the repeated electrophysiological studies. Athough increased risk of thrombosis has been previously reported [31,32] during lenalidomide treatment, in our study, no thrombotic events were observed. The results need to be interpreted with caution since none of the patients had other associated cardiovascular risk factors, such as smoking or presence of antiphospholipid antibodies.

Still not clear is the effect of lenalidomide on systemic disease. Braunstein et al. described an increasing risk of developing a severe systemic lupus flare in their small cohort of patients with one treated patient developing a renal flare. They attributed it to the possible effect of lenalidomide on $\mathrm{T}$ cell activation and pDCs [24]. However, no 
Table 3 Adverse events reported during lenalidomide treatment.

\begin{tabular}{llll}
\hline Patient & Graded diagnosis & NCl toxicity grade & Causal relationship to lenalidomide treatment \\
\hline 3 & Vomiting & Grade 2 & Possible \\
& Diarrhea & Grade 2 & Possible \\
& Weight loss & Grade 2 & Possible \\
& Fatigue & Grade 2 & Possible \\
15 & Insomnia & Grade 2 & Probable \\
& Neutropenia & & Possible \\
\hline
\end{tabular}

significant clinical or immunological effect of lenalidomide on systemic manifestations of SLE was observed in our larger cohort of treated patients after 15 months of follow-up. Only one patient with known systemic disease developed mild arthritis. None of the patients with CLE progressed to a systemic disease and levels of antidsDNA antibodies or complement remained stable during follow-up.

Results were compared with those obtained in our previous cohort of thalidomide-treated patients [17]. Although lenalidomide was better tolerated and had a safer profile, both agents displayed the same efficacy and relapse rates. As lenalidomide is an analogue of thalidomide, the question of resistance to lenalidomide in patients unresponsive to thalidomide arose. Both the literature [21] and our study indicate that clinical benefit from lenalidomide can be obtained in those refractory patients, reflecting mechanistic differences - lenalidomide has greater immunomodulatory properties than thalidomide, whereas thalidomide has greater antiangiogenic activity [20]. Lenalidomide has been found to be more potent in the stimulation of T-cell proliferation and IFNgamma and IL-2 production than thalidomide, whereas both thalidomide and pomalidomide, another thalidomide analogue, have been found to be more potent at inhibiting sprout formation than lenalidomide when antiangiogenic properties were assessed in a human umbilical explant model (20).

The main limitations of the study are the absence of a randomized group control and the insufficient small sample size to draw conclusions at the histological subtype level since the majority of included patients had DLE. Nevertheless, the present study provides valuable information on the critical issue of treating refractory cutaneous lupus lesions given the significant number of patients studied, the relatively long period of follow-up and the detailed clinical and laboratory information obtained during the follow-up. Further larger controlled trials are required to confirm our observations.

\section{Conclusions}

In conclusion, the present study confirms the efficacy and safety of lenalidomide for refractory cutaneous lupus disease. The benefit of lenalidomide is in reducing disfigurement without thalidomide's toxicity profile.

\section{Abbreviations}

ACLE: acute cutaneous lupus erythematosus; ACR: American College of Rheumatology; AE: adverse event; ANA: antinuclear antibodies; CLASI: Cutaneous Lupus Erythematosus Disease Area and Severity Index; CLE: cutaneous lupus erythematosus; CR: complete response; DLE: discoid lupus erythematosus; ENA: extractable nuclear antigen; LET: lupus erythematosus tumidus; PR: partial response; SAE: serious adverse event; SCLE: subacute cutaneous lupus erythematosus; SLE: systemic lupus erythematosus; SLEDAl: Systemic Lupus Erythematosus Disease Activity Index.

\section{Acknowledgements}

This project has been supported by the Spanish Ministry of Health: TRA-194/ 2009.

\section{Authors' contributions}

$\mathrm{JCH}$ participated in the design of the study, collected the data, performed data analysis/interpretation and drafted the manuscript. GA participated in data analysis and drafted the manuscript. MVT participated in data interpretation and edited the manuscript. JOR conceived and participated in the design of the study, data interpretation and draft of the manuscript. All authors read and approved the final manuscript

\section{Authors' information}

GA is a rheumatologist at the Lupus Unit at the Department of Autoimmune Diseases, Internal Medicine, University Autónoma of Barcelona, Spain. JCH is a lecturer at the Lupus Unit, Department of Autoimmune Diseases, Internal Medicine, University Autónoma of Barcelona, Spain. MVT is Professor and director at the Department of Internal Medicine, Faculty of Medicine, University Autónoma of Barcelona, Spain. JOR is Professor and director of the Lupus Unit at the Department of Internal Medicine, Faculty of Medicine, University Autónoma of Barcelona, Spain.

\section{Competing interests}

The authors declare that they have no competing interests.

Received: 23 July 2012 Revised: 29 September 2012

Accepted: 12 November 2012 Published: 7 December 2012

\section{References}

1. Lee HJ, Sinha AA: Cutaneous lupus erythematosus: understanding of clinical features, genetic basis, and pathobiology of disease guides therapeutic strategies. Autoimmunity 2006, 39:433-444.

2. Gilliam JN, Sontheimer RD: Distinctive cutaneous subsets in the spectrum of lupus erythematosus. J Am Acad Dermatol 1981, 4:471-475.

3. Crowson AN, Magro C: The cutaneous pathology of lupus erythematosus: a review. J Cutan Pathol 2001, 28:1-23.

4. Kuhn A, Ruland V, Bonsmann G: Cutaneous lupus erythematosus: update of therapeutic options Part I. J Am Acad Dermatol 2011, 65:e179-193.

5. Callen JP: Management of 'refractory' skin disease in patients with lupus erythematosus. Best Pract Res Clin Rheumatol 2005, 19:767-784.

6. Kuhn A, Ruland V, Bonsmann G: Cutaneous lupus erythematosus: update of therapeutic options Part II. J Am Acad Dermatol 2011, 65:e195-213. 
7. Mellin GW, Katzenstein M: The saga of thalidomide. Neuropathy to embriopathy, with case reports of congenital abnormalities. N Engl J Med 1962, 267:1184-1192.

8. Wu JJ, Huang DB, Pang KR, Hsu S, Tyring SK: Thalidomide: dermatological indications, mechanisms of action and side-effects. Br J Dermatol 2005, 153:254-273

9. Housman TS, Jorizzo JL, McCarty MA, Grummer SE, Fleischer AB Jr, Sutej PG: Low-dose thalidomide therapy for refractory cutaneous lesions of lupus erythematosus. Arch Dermatol 2003, 139:50-54.

10. Kyriakis KP, Kontochristopoulus GJ, Panteleos DN: Experience with lowdose thalidomide therapy in chronic discoid lupus erythematosus. Int J Dermatol 2000, 39:218-222.

11. Knop J, Bonsmann G, Happle R, Ludolph A, Matz DR, Mifsud EJ, Macher E: Thalidomide in the treatment of sixty cases of chronic discoid lupus erythematosus. Br J Dermatol 1983, 108:461-466.

12. Sato El, Assis LS, Lourenzi VP, Andrade LE: Long-term thalidomide use in refractory cutaneous lesions of systemic lupus erythematosus. Rev Assoc Med Bras 1998, 44:289-293.

13. Ordi-Ros J, Cortés F, Cucurull E, Mauri M, Buján S, Vilardell M: Thalidomide in the treatment of cutaneous lupus refractory to conventional therapy. J Rheumatol 2000, 27:1429-1433.

14. Stevens RJ, Andujar C, Edwards CJ, Ames PR, Barwick AR, Khamashta MA, Hughes GR: Thalidomide in the treatment of the cutaneous manifestations of lupus erythematosus: experience in sixteen consecutive patients. Br J Rheumatol 1997, 36:353-359.

15. Coelho A, Souto MID, Cardoso CR, Salgado DR, Schmal TR, Waddington Cruz M, de Souza Papi JA: Long-term thalidomide use in refractory cutaneous lesions of lupus erythematosus: a 65 series of Brazilian patients. Lupus 2005, 14:434-439.

16. Cuadrado MJ, Karim Y, Sanna G, Smith E, Khamashta MA, Hughes GR: Thalidomide for the treatment of resistant cutaneous lupus: efficacy and safety of different therapeutic regimens. Am J Med 2005, 118:246-250.

17. Cortés-Hernández J, Torres-Salido M, Castro-Marrero J, Vilardell-Tarres M, Ordi-Ros J: Thalidomide in the treatment of refractory cutaneous lupus: prognostic factors of clinical outcome. Br J Dermatol 2012, 166:616-623.

18. List A, Dewald G, Bennett J, Giagounidis A, Raza A, Feldman E, Powell B, Greenberg P, Thomas D, Stone R, Reeder C, Wride K, Patin J, Schmidt M: Lenalidomide in the myelodysplastic syndrome with chromosome $5 q$ deletion. N Engl J Med 2006, 355:1456-1465

19. Richardson PG, Blood E, Mitsiades CS, Jagannath S, Zeldenrust SR, Alsina M, Schlossman RL, Rajkumar SV, Desikan KR, Hideshima T, Munshi NC, KellyColson K, Doss D, McKenney ML, Gorelik S, Warren D, Freeman A, Rich R, Wu A, Olesnyckyj M, Wride K, Dalton WS, Zeldis J, Knight R, Weller E, Anderson KC: A randomized phase 2 study of lenalidomide therapy for patients with relapsed or relapsed and refractory multiple myeloma. Blood 2006, 108:3458-3464.

20. Anderson KC: Lenalidomide and thalidomide: mechanisms of action similarities and differences. Semin Hematol 2005, 42:53-8.

21. Wang M, Dimopoulos MA, Chen C, Cibeira MT, Attal M, Spencer A, Rajkumar SV, Yu Z, Olesnyckyj M, Zeldis JB, Knight RD, Weber DM: Lenalidomide plus dexamethasone is more effective than dexamethasone alone in patients with relapsed or refractory multiple myeloma regardless of prior thalidomide exposure. Blood 2008, 112:4445-4451.

22. Sharma RA, Steward WP, Daines CA, Knight RD, O'Byrne KJ, Dalgleish AG: Toxicity profile of the immunomodulatory thalidomide analogue, Lenalidomide: phase I clinical trial of three dosing schedules in patients with solid malignancies. Eur J Cancer 2006, 42:2318-2325.

23. Shah A, Albrecht J, Bonilla-Martinez Z, Okawa J, Rose M, Rosenbach M, Werth VP: Lenalidomide for the treatment of resistant discoid lupus erythematosus. Arch Dermatol 2009, 145:303-306.

24. Braunstein I, Goodman NG, Rosenbach M, Okawa J, Shah A, Krathen M, Kovarik CL, Luning Prak E, Werth VP: Lenalidomide therapy in treatmentrefractory cutaneous lupus erythematosus: Histologic and circulating leukocyte profile and potential risk of a systemic lupus flare. J Am Acad Dermatol 2012, 66:571-582.

25. Albrecht J, Taylor L, Berlin JA, Dulay S, Ang G, Fakharzadeh S, Kantor J, Kim E, Militello G, McGinnis K, Richardson S, Treat J, Vittorio C, Van Voorhees A, Werth VP: The CLASI (Cutaneous Lupus Erythematosus Disease Area and Severity Index): an outcome instrument for cutaneous lupus erythematosus. J Invest Dermatol 2005, 125:889-894.
26. Gladman DD, Ibañez D, Urowitz MB: Systemic lupus erythematosus disease activity index 2000. J Rheumatol 2002, 29:288-291.

27. Tan EM, Cohen AS, Fries JF, Masi AT, McShane DJ, Rothfield NF, Schaller JG, Talal N, Winchester RJ: The 1982 revisited criteria for the classification of systemic lupus erythematosus. Arthritis Rheum 1982, 25:1271-1277.

28. Jessop S, Whitelaw DA, Delamare FM: Drugs for discoid lupus erythematosus. Cochrane Database Syst Rev 2009, 4:CD002954.

29. Tzung TY, Liu YS, Chang HW: Tacrolimus vs. clobetasol propionate in the treatment of facial cutaneous lupus erythematosus: a randomized, double-blind, bilateral comparison study. Br J Dermatol 2007, 156:191-192.

30. Jemec GB, Ullman S, Goodfield M, Bygum A, Olesen AB, Berth-Jones J, Nyberg F, Cramers M, Faergemann J, Andersen P, Kuhn A, Ruzicka T: A randomized controlled trial of R-salbutamol for topical treatment of discoid lupus erythematosus. Br J Dermatol 2009, 161:1365-1370.

31. Barlett JB, Michel A, Clarke IA, Dredge K, Nicholson S, Kristeleit H, Polychronis A, Pandha H, Muller GW, Stirling DI, Zeldis J, Dalgleish AG: Phase I study to determine the safety, tolerability and immunostimulatory activity of lenalidomide analogue CC-503 in patients with metastatic malignant melanoma and other advanced cancers. $\mathrm{Br}$ J Cancer 2004, 90:955-961.

32. Warren KE, Goldman S, Pollack IF, Fangusaro J, Schaiquevich P, Stewart CF, Wallace D, Blaney SM, Packer R, Macdonald T, Jakacki R, Boyett JM, Kun LE: Phase I trial of Lenalidomide in pediatric patients with recurrent, refractory, or progressive primary CNS tumors: Pediatric Brain Tumor consortium Study PBTC-018. J Clin Oncol 2011, 29:324-329.

doi:10.1186/ar4111

Cite this article as: Cortés-Hernández et al.: Efficacy and safety of lenalidomide for refractory cutaneous lupus erythematosus. Arthritis Research \& Therapy 2012 14:R265.

\section{Submit your next manuscript to BioMed Central and take full advantage of:}

- Convenient online submission

- Thorough peer review

- No space constraints or color figure charges

- Immediate publication on acceptance

- Inclusion in PubMed, CAS, Scopus and Google Scholar

- Research which is freely available for redistribution

Submit your manuscript at www.biomedcentral.com/submit
C Biomed Central 